\title{
Densidad Neuronal en la Corteza Visual Primaria (Área 17), en Ratas Sometidas a Estrés Crónico
}

\author{
Density in Primary Visual Cortex (17 Visual Area) from Rats Subjected to Chronic Stress
}

\author{
"Olivares, R.; "Toledo, C.; "Vera, Y.; *"Morgan, C.; "*Pérez, H.; \\ ****íz, A.; ${ }^{* * *}$ Henríquez, M.; ${ }^{* * * * *}$ Hernández, A. \& ${ }^{* * * * * *}$ Cifuentes, F.
}

OLIVARES, R.; TOLEDO, C.; VERA, Y.; MORGAN, C.; PÉREZ, H.; ORTÍZ, A.; HENRÍQUEZ, M.; HERNÁNDEZ, A. \& CIFUENTES, F. Densidad neuronal en la corteza visual primaria (área 17) en ratas sometidas a estrés crónico. Int. J. Morphol., 28(3):855-860, 2010.

RESUMEN: El estrés puede ser definido como una amenaza a la integridad psicológica o fisiológica de un individuo. Por otro lado, se ha verificado que el estrés tiene efecto sobre la morfología y función de diversas estructuras del Sistema Nervioso Central, relacionadas con el aprendizaje, memoria y respuestas emocionales, tales como el hipocampo, amígdala y corteza prefrontal. Es por lo anterior, que el objetivo del presente trabajo fue realizar un estudio de la anatomía de la corteza visual primaria (área 17), en ratas machos (n=9), de la cepa Sprague-Dawley, de 3 meses de edad (250-350g.), sometidas a estrés crónico por inmovilización. Es así como se observó que el grupo estrés $(n=3)$ presentó una menor densidad neuronal que el grupo control ( $\mathrm{n}=3$ ) y una significativa menor densidad neuronal $(\mathrm{p}<0,05)$ que el grupo postestrés $(n=3)$ el cual presentó la más alta densidad neuronal observada. Estableciendo una relación inversa entre densidad neuronal y tamaño de los somas neuronales y sus respectivas conexiones y ramificaciones dendríticas. Lo anterior podría tener incidencia en el procesamiento de la información visual.

PALABRAS CLAVE: Estrés; Corteza Visual; Densidad neuronal; Rata.

\section{INTRODUCCIÓN}

El estrés, se define como una respuesta general del organismo antes demandas externas o internas, inicialmente amenazantes, que consiste en movilizar recursos fisiológicos y psicológicos para poderlas afrontar. Es decir, será el desequilibrio entre las demandas del organismo y la capacidad de éste para sobrellevarlas, lo que caracteriza una situación estresante, el mantenimiento de esta situación caracteriza al estrés crónico o diestrés (Montoro et al., 2009).

El estrés tiene un profundo efecto sobre la morfología y función de diversas estructuras del Sistema Nervioso Central. En ratas, se ha observado que los protocolos de estrés inducen alteraciones morfológicas en hipocampo, amígdala y corteza prefrontal, estructuras que están relacionadas con el aprendizaje, memoria y respuestas emocionales (Vyas et al., 2002).
En humanos con depresión, estudios de neuroimágenes han evidenciado atrofia del volumen hipocampal, reducción de la sustancia gris y blanca en la corteza prefrontal y disminución del volumen amigdalino (Sheline et al., 1996; McEwen \& Chattarji, 2004). Estudios postmortem en cerebros de pacientes con depresión mayor, mostraron reducción del tamaño neuronal y/o disminución de la densidad de las células gliales en la corteza orbitofrontal, dorsolateral y corteza prefrontal (Rajkowska et al., 1999; Manji et al., 2003).

Adicionalmente, se ha demostrado el rol del estrés prenatal, sobre eventuales alteraciones en la morfología cerebral y en funciones cognitivas, emocionales y conductuales (Lemaire et al., 2000). En los últimos años, numerosos estudios indican que largos periodos de estrés afectan la plas-

* Departamento de Ciencias Biológicas Animales, Facultad de Ciencias Veterinarias y Pecuarias, Universidad de Chile.

* Laboratorio de Nutrición y Regulación Metabólica, Instituto de Nutrición y Tecnología de los Alimentos (INTA), Universidad de Chile.

**** Escuela de Medicina Veterinaria, Facultad de Ecología y Recursos Naturales, Universidad Nacional Andrés Bello.

**** Departamento de Biología, Facultad de Química y Biología, Universidad de Santiago de Chile.

******* Departamento de Patología Animal, Facultad de Ciencias Veterinarias y Pecuarias, Universidad de Chile.

Financiamiento: Fondo de Investigación Veterinaria (FIV) No 121014019102004 
ticidad sináptica, la morfología dendrítica y la neurogénesis (Weinstock, 2001). En esta línea, se ha observado que en ratas adultas, la malnutrición proteica prenatal es capaz de alterar las concentraciones extracelulares de dopamina y serotonina en la corteza prefrontal medial y dorsal del hipocampo, cuando dichos animales son además sometidos a un estrés por restricción (Mockler et al., 2007).

Sobre la base de los antecedentes expuestos, el objetivo del presente trabajo fue evaluar en ratas, los efectos de un estrés crónico por inmovilización y de un periodo de recuperación postestrés, sobre la anatomía de la corteza visual primaria (área 17).

\section{MATERIAL Y MÉTODO}

Animales y protocolo de estrés por inmovilización. Ratas machos de la cepa Sprague-Dawley de 3 meses de edad (250$350 \mathrm{~g}$.), fueron asignadas en forma aleatoria en un grupo Control A, un grupo Estrés B y un grupo Postestrés C, de 3 animales cada uno. Los animales de los grupos B y C, fueron sometidos a inmovilización por 10 días ( 2 horas por día, de las 10 a $12 \mathrm{~h}$.), de acuerdo al protocolo descrito por Vyas et al., el grupo $\mathrm{C}$ fue sacrificado 15 días después de haber terminado el periodo de estrés. El manejo experimental, se realizó en el Bioterio del INTA de la Universidad de Chile, bajo un régimen de 12 horas luz/12 horas oscuridad a una temperatura ambiente de $21 \pm 1.2^{\circ} \mathrm{C}$ y con acceso ad libitum a agua y comida. El protocolo de experimentación y método de sacrificio de los animales fue aprobado por el Comité de Bioética Animal de la Facultad de Ciencias Veterinarias y Pecuarias de la Universidad de Chile.

Las cajas de inmovilización presentaron una dimensión de 18x6x6cm., que permitió una completa restricción del animal, pero con un espacio mínimo necesario para que eventualmente éstos pudieran defecar y orinar y no tener contacto con sus deshechos. Los animales fueron pesados diariamente y al momento de su sacrificio, fueron anestesiados con Tiopental Sódico (40mg/Kg i.p.) y luego perfundidos transcardialmente con formalina al 5\%, posteriormente los cuerpos fueron decapitados y las cabezas descalotadas, para extraer los encéfalos y luego los cerebros fueron fijados en formalina al $10 \%$. Adicionalmente, en todos los grupos, se calculó el porcentaje de gananciade peso (peso final/peso inicial x 100), el peso relativo de las glándulas adrenales (peso glándulas/peso cuerpo x 100) y se verificó la presencia de úlceras en la mucosa gástrica (Brzozowski et al., 2000).

Estudio de la corteza visual primaria (área 17), mediante microscopía de luz, con la técnica de la celoidina
Los cerebros ( $\mathrm{n}=9$ ), fueron lavados en agua corriente por un período de 24 horas para luego ser incluidos en celoidina de acuerdo a la técnica descrita por Yakovlev (1970). Una vez impregnados los encéfalos en celoidina y endurecido el material, se realizaron cortes coronales anteroposteriores, de $40 \mathrm{~mm}$ de espesor, los que fueron teñidos con Cresil Violeta (Nissl) al 1\%, a fin de poder visualizar con mayor facilidad los somas neuronianos (Soto-Moyano et al., 1999). Se seleccionaron los cortes correspondientes a la región dorsomedial del área 17 y, dentro de éstos, las capas IV y V, tomando como referencia un mapa estereotáxico de la citoarquitectura de la corteza cerebral presente en roedor (Caviness, 1975) y la histología del área 17 de la corteza cerebral de rata (Peters \& Kara, 1985a, 1985b).

La densidad neuronal fue cuantificada, mediante la utilización de la técnica del disector óptico (Braendgaard et al., 1990). El conteo se realizó en las capas IV y V y se contabilizaron principalmente neuronas piramidales que se encontraban en el mismo plano focal y se descartaron las que se encontraban en el plano más superficial de la sección y las que interceptaban dos planos contiguos (West \& Gundersen, 1990). El conteo se realizó con el aumento mayor del microscopio (100x), en un volumen tisular de $64.000 \mu \mathrm{m}^{3}$. La densidad se expresó finalmente en neuronas $/ \mathrm{mm}^{3}$. El procedimiento se realizó siempre en el mismo hemisferio cerebral y aproximadamente en el mismo punto, a fin de evitar posibles efectos de lateralización que pudiesen sesgar los resultados (Soto-Moyano et al.). En cada cerebro se trabajó con un promedio de 21 cortes y en cada uno de ellos se efectuaron 4 mediciones distintas, las que se promediaron para obtener un valor de densidad por corte.

Análisis estadístico. Los valores de la densidad neuronal del área 17 de la corteza cerebral; para cada uno de los grupos en estudio, fueron corregidos a su magnificación real, tabulados y caracterizados (promedio y desviación estándar), para su análisis estadístico mediante una prueba de ANDEVA no paramétrica (Kruskal-Wallis) y una prueba de comparación múltiple (Dunn).

\section{RESULTADOS}

La densidad neuronal de la corteza primaria se puede observar en la Tabla I. Las letras distintas señalan diferencias estadísticamente significativas (Dunn $\mathrm{p}<0,05$ ), de tal forma que "a" (Grupo Estrés) es diferente de "b" (Grupo Postestrés) y "ab" (Grupo Control) no es diferente ni de "a" ni de "b". 
En la Figura 1 se observan los somas de neuronas piramidales de la corteza visual primaria (Área 17), de las capas IV y V. A) Grupo Control, B) Grupo Estrés y C) Grupo Postestrés.

Tabla I. Densidad neuronal de la corteza visual primaria (Área 17).

\begin{tabular}{|c|c|c|c|}
\hline neuronas $/ \mathrm{mm}^{3}$ & Grupo Control $(n=3)$ & Grupo Estrés (n=3) & Grupo Postestrés $(n=3)$ \\
\hline $\mathrm{p}=0,0107 \mathrm{~K}-\mathrm{W}=6,49$ & $42,46 \pm 2,13 \times 10^{4} \mathrm{ab}$ & $39,72 \pm 0,92 \times 10^{4} \quad a$ & $44,67 \pm 2,33 \times 10^{4} \quad b$ \\
\hline
\end{tabular}
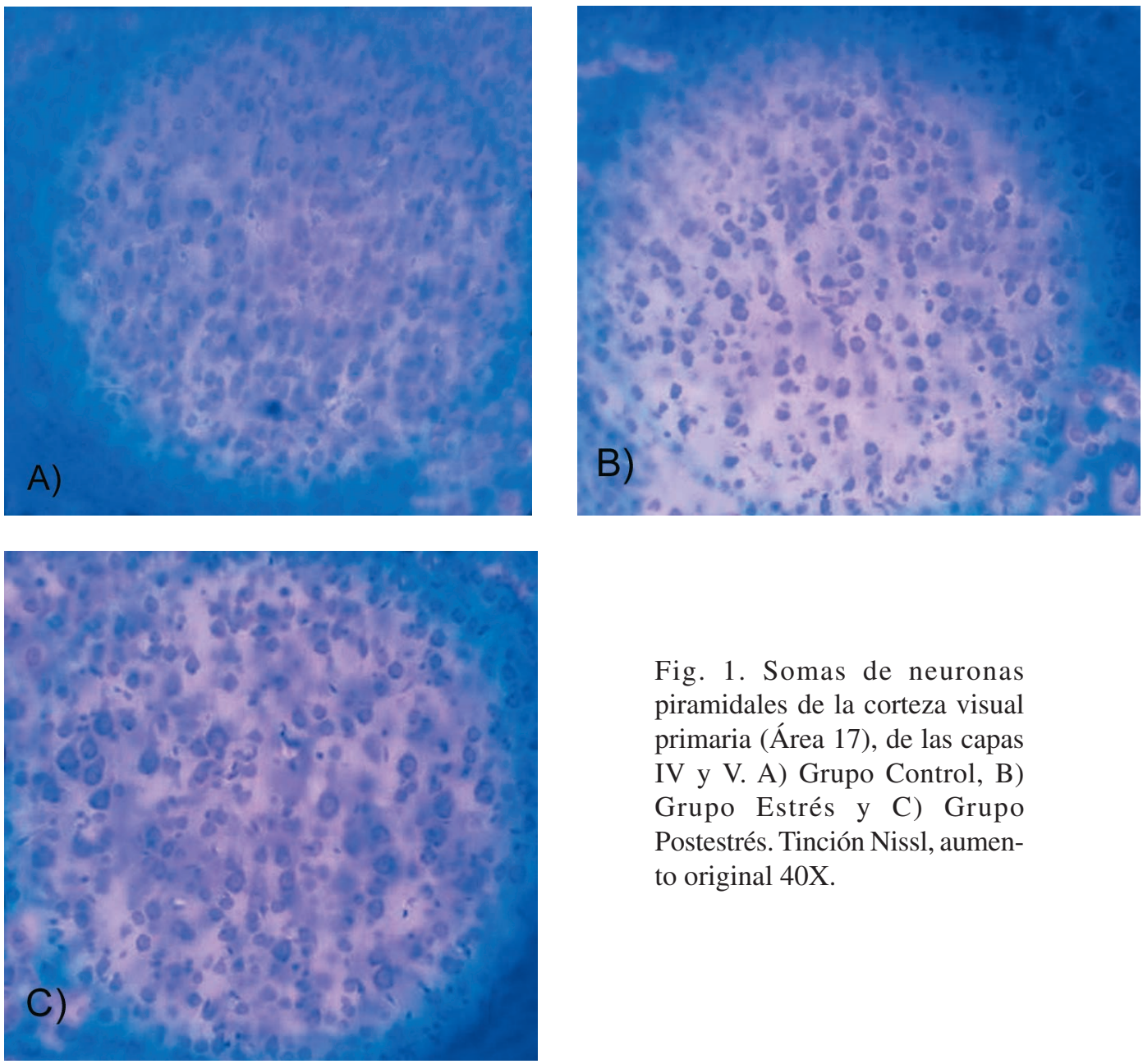

Fig. 1. Somas de neuronas piramidales de la corteza visual primaria (Área 17), de las capas IV y V. A) Grupo Control, B) Grupo Estrés y C) Grupo Postestrés. Tinción Nissl, aumento original $40 \mathrm{X}$.
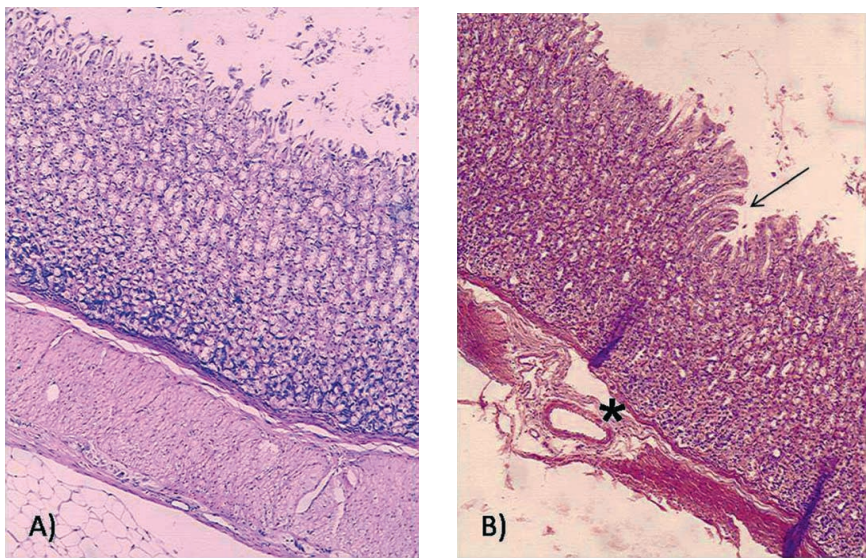

Fig. 2. Cambios en la mucosa gástrica en ratas sometidas a estrés. El grupo control (A), no presenta lesiones; el grupo estrés (B), presenta erosión superficial de la mucosa (Flecha) y edema leve de la submucosa (asterisco). Tinción H-E, aumento original $100 \mathrm{X}$. 
Complementariamente, se analizaron los efectos del estrés por inmovilización, sobre la morfología de la mucosa gástrica, el peso corporal y de las glándulas adrenales. Se observó erosión de la mucosa gástrica (Figura 2); una diferencia significativa $(\mathrm{p}<0,0001)$, en el porcentaje de ganancia de peso (Control=7,34 $\pm 3,58 \%$; Estrés $=1,93 \pm 3,57 \%$; Postestrés $=16,23 \pm 5,61 \%)$; y en el peso relativo de las glándulas adrenales $(\mathrm{p}<0,05),($ Control $=0,013 \pm 0,002 \%$; Estrés $=0,017 \pm 0,003 \%$; Postestrés $=0,015 \pm 0,002 \%$ ), respectivamente. Estos resultados indicarían que el protocolo de estrés, fue aplicado correctamente (Dagnino-Subiabre et al., 2005).

\section{DISCUSIÓN}

El patrón altamente organizado de las conexiones córtico-corticales, que se observa en el animal adulto, representaría el estadio final de un proceso de desarrollo que reconfigura las conexiones dispersas, inmaduras, presentes en el neonato (Bravo \& Inzunza, 1994).

Existen evidencias experimentales, fundamentalmente en roedores, que demuestran que la organización final del sistema nervioso depende de factores no genéticos, los que actúan en el período prenatal y postnatal temprano (SotoMoyano et al.). Es así como, el deterioro medioambiental durante un período limitado de tiempo (desde el nacimiento hasta los 18 días postnatales), produce una disminución de las ramificaciones dendríticas de las neuronas de la corteza motora de la rata, lo que a su vez se puede ver potenciado combinando lo anterior con una desnutrición (Fernández et al., 1993).

Las condiciones naturales de luz y oscuridad en que se desenvuelven las diferentes especies animales, influirían en el desarrollo de la corteza cerebral occipital, zona destinada al procesamiento de la información relativa a la visión. Es así como, se ha observado en roedores silvestres, con diferentes períodos de actividad, variaciones en la densidad neuronal cortical occipital (Olivares et al., 2004). Recientemente, Olivares et al. (2010), compararon especies más cercanas filogenéticamente, de manera de minimizar la variable taxonómica. En este estudio se evidenció que Octodon degus, el cual presenta un período de actividad diurna, mostraba una densidad neuronal menor en la corteza visual primaria (área 17), que la observada en $O$. bridgesi, especie de período de actividad nocturna; lo cual fue estadísticamente significativo.

En otra línea, ratas sometidas a una dieta hipoproteica-isocalórica, desde la gestación hasta los 22 días de edad, presentaron en la corteza cerebral occipital una densidad neuronal mayor que los animales controles y los rehabilitados durante la lactancia (Soto-Moyano et al.).

Estudios en animales que son sometidos a un estrés crónico y hormonal, han demostrado que el estrés induce remodelación dendrítica de las neuronas piramidales del área CA3 del hipocampo, disminuyendo en el adulto la neurogénesis en el giro dentado y reduciendo el volumen hipocampal total. Siendo estas alteraciones reversibles postestrés (Czéh et al., 2001).

Ratas que fueron sometidas a un estrés por inmovilización, presentaron atrofia dendrítica en las neuronas del colículo caudal, estructura relacionada con la vía auditiva refleja y al dar un periodo de recuperación de 15 días postestrés, las neuronas de dicho colículo, presentaron su estructura dendrítica totalmente restaurada (DagninoSubiabre et al.).

Por último se puede señalar, que se ha estudiado el efecto del estrés crónico por inmovilización, sobre el desarrollo dentrítico del cerebro anterior, en fetos de ratas preñadas. El análisis morfométrico estereológico demostró que los fetos de 12 días de gestación de madres estresadas, evidenciaron un aumento del tamaño de la vesícula telencefálica. El perímetro y el espesor del cerebro anterior, mostraron diferencias significativas en relación a los controles, pero no así en su forma. Este efecto del estrés crónico se podría considerar reversible en los estadíos gestacionales subsecuentes (Mugnaini et al., 2006).

En relación a la medición de la densidad neuronal de la corteza visual primaria (área 17), observamos en este trabajo, que el grupo estrés presentó una menor densidad que el control y que el grupo postestrés, siendo esto último estadísticamente significativo $(\mathrm{p}<0,05)$; además, el grupo postestrés presentó una mayor densidad neuronal que el grupo control (Tabla I y Fig. 1). Estableciendo una relación inversa entre densidad neuronal y tamaño de los somas neuronales y sus respectivas conexiones y ramificaciones dendríticas (Soto-Moyano et al.). Lo anterior podría tener incidencia en la transferencia interhemisférica y subcortical, en especial en lo que dice relación con la información visual; debiendo ser corroborado a futuro, analizando un mayor número de animales como también otras áreas corticales.

\section{AGRADECIMIENTOS}

Dr. Francisco Aboitiz D. Laboratorio de Neurobiología, Centro de Investigaciones Médicas, Pontificia Universidad Católica de Chile. 
OLIVARES, R.; TOLEDO, C.; VERA, Y.; MORGAN, C.; PÉREZ, H.; ORTÍZ, A.; HENRÍQUEZ, M.; HERNÁNDEZ, A. \& CIFUENTES, F. Density in primary visual cortex (17 visual area) from rats subjected to chronic stress. Int. J. Morphol., 28(3):855$860,2010$.

SUMMARY: Stress can be understood as a threat to psychological or physiological integrity of the individual. Stress has previously shown to alter morphology and function of diverse structures of the Central Nervous System related to learning, memory and emotional response, such as hippocampus, amygdala and prefrontal cortex. In the current work we assessed the effect of chronic stress for immobilization on structure of primary visual cortex (area 17) in male adult Sprague-Dawley rats ( $\mathrm{n}=9$ ), of 3 months of age (250-350g.). Stressed rats $(n=3)$ tended to show lower neuronal densities than control rats $(n=3)$ and were significantly lower $(p<0.05)$ than recovered post-stress rats $(n=3)$, which showed the highest neuronal densities observed. Since an inverse correlation between neuronal density and size of neuronal bodies and their respective dendrite branches, these changes might impact processing of visual information.

KEY WORDS: Stress; Visual cortex; Neuronal density; Rat.

\section{REFERENCIAS BIBLIOGRÁFICAS}

Braendgaard, H.; Evans, S. M.; Howard, C. V. \& Gundersen, H. J. G. The total number of neurons in the human neocortex unbiasedly estimated using optical dissector. J. Microsc., 157:285-304, 1990.

Bravo, H. \& Inzunza, O. Effects of pre and postnatal retinal deprivation on the striate-peristriate cortical connections in the rat. Biol. Res., 27:73-7, 1994.

Brzozowski, T.; Konturek, P. C.; Konturek, S. J.; Drozdowicz, D.; Kwiecien, S.; Pardo, R.; Bielanski, W. \& Hahn, E. G. Role of gastric acid secretion in progresión of acute gastric erosions induced by ischemiareperfusion into gastric ulcers. Eur. J. Pharmacol., 398:147-58, 2000.

Caviness, V. S. Jr. Architectonic map of neocortex of the normal mouse. J. Comp. Neurol., 164:247-64, 1975.

Czéh, B.; Michaelis, T.; Watanabe, T.; Frahm, J.; de Biurrun, G.; van Kampen, M.; Bartolomucci, A. \& Fuchs, E. Stress-induced changes in cerebral metabolites, hippocampal volume, and cell proliferation are prevented by antidepressant treatment with tianeptine. Proc. Natl. Acad. Sci. USA, 98:12796-801, 2001.

Dagnino-Subiabre, A.; Terreros, G.; Carmona-Fontaine, C.; Zepeda, R.; Orellana, J. A.; Díaz-Véliz, G.; Mora, S. \& Aboitiz, F. Chronic stress impairs acoustic conditioning more than visual conditioning in rats: Morphological and behavioural evidence. Neuroscience, 135:1067-74, 2005.

Fernández, V.; Pascual, R. \& Ruiz, S. Early - life environmental deterioration, nutrition and ontogenesis of the motor cortex in the rat: a Golgi study. Biol. Neonate, 64:245-53, 1993.
Lemaire, V.; Koehl, M.; Le Moal, M. \& Abrous, D. N. Prenatal stress produces learning deficits associated with an inhibition of neurogenesis in the hippocampus. Proc. Natl. Acad. Sci. USA, 97: 11032-7, 2000.

Manji, H. K.; Quiroz, J. A.; Sporn, J.; Payne, J. L.; Denicoff, K.; Gray, N. A.; Zarate, C. A. Jr. \& Charney, D. S. Enhancing neuronal plasticity and cellular resilience to develop novel, improved therapeutics for difficult-totreat depression. Biol. Psychiatry, 53:707-42, 2003.

McEwen, B. S. \& Chattarji, S. Molecular mechanisms of neuroplasticity and pharmacological implications: the example of tianeptine. Eur. Neuropsychopharmacol., 14(5):S497-502, 2004.

Mockler, D. J.; Torres, O. I.; Galler, J. R. \& Morgane, P. J. Stress-induced changes in extracellular dopamine and serotonin in the medial prefrontal cortex and dorsal hippocampus of prenatally malnourished rats. Brain Res., 1148:226-33, 2007.

Montoro, J.; Mullol, J.; Jáuregui, I.; Dávila, I.; Ferrer, M.; Bartra, J.; Del Cuvillo, A.; Sastre, J. \& Valero, A. Stress and allergy. J. Investig. Allergol. Clin. Immunol., 19:40-7, 2009.

Mugnaini, M. T.; Soñez, C. A.; Rolando, A. N.; Romanini, M. C.; Bozzo, A. A.; Pastorino, I. C.; Gauna, H. F. \& Paz, D. A. Maternal chronic stress induces premature telencephalic vesicles development. Int. J. Morphol., 24:525-30, 2006.

Olivares, R.; Godoy, G.; Adaro, L. \& Aboitiz, F. Densidad neuronal de la corteza visual primaria (área 17), de dos especies de roedores silvestres. Int. J. Morphol., 22:27984, 2004. 
OLIVARES, R.; TOLEDO, C.; VERA, Y.; MORGAN, C.; PÉREZ, H.; ORTÍZ, A.; HENRÍQUEZ, M.; HERNÁNDEZ, A. \& CIFUENTES, F. Densidad neuronal en la corteza visual primaria (área 17) en ratas sometidas a estrés crónico. Int. J. Morphol., 28(3):855-860, 2010.

Olivares, R.; Ortíz, A.; Henríquez, M.; Adaro, L. \& Aboitiz, F. Densidad neuronal en la corteza visual primaria (Area 17), en dos especies de Octodon. Int. J. Morphol., 28:249-53, 2010.

Peters, A. \& Kara, D. The neuronal composition of area 17 of rat visual cortex. I. The pyramidal cells. J. Comp. Neurol., 234:218-41, 1985a.

Peters, A. \& Kara, D. The neuronal composition of area 17 of rat visual cortex. II. The nonpyramidal cells. J. Comp. Neurol., 234:241-63, 1985b.

Rajkowska, G.; Miguel-Hidalgo, J. J.; Wei, J.; Dilley, G.; Pittman, S. D. \& Meltzer, H. Y. Morphometric evidence for neuronal and glial prefrontal cell pathology in major depression. Biol. Psychiatry, 45:1085-98, 1999.

Sheline, Y. I.; Wang, P. W.; Gado, M. H.; Csernansky, J. G. \& Vannier, M. W. Hippocampal atrophy in recurrent major depression. Proc. Natl. Acad. Sci. USA, 93:390813, 1996.

Soto-Moyano, R.; Fernández, V.; Sanhueza, M.; Belmar, J.; Kusch, C.; Pérez, H.; Ruiz, S. \& Hernández, A. Effects of mild protein prenatal malnutrition and subsequent postnatal nutritional rehabilitation on noradrenalina release and neuronal density in the rat occipital cortex. Dev. Brain Res., 116:51-8, 1999.

Vyas, A.; Mitra, R.; Shakaranarayana Rao, B. S. \& Chattarji, S. Chronic stress induces contrasting patterns of dendritic remodeling in hippocampal and amygdaloid neurons. $J$. Neurosci., 22:6810-8, 2002.

Weinstock, M. Alterations induced by gestational stress in brain morphology and behavior of the offsprings. Progress Neurobiol., 65:427-51, 2001.

West, M. \& Gundersen, H. Unbiased stereological estimation of the number of neurons in the human hippocampus. $J$. Comp. Neurol., 296:1-22, 1990.

Yakovlev, B. Whole brain serial histological sections. In: Tedeschi, C. G. (Ed.). Neuropathology, Methods and Diagnosis. Boston, Little, Brown and Co., 1970. pp.3718.
Dirección para correspondencia:

Ricardo Olivares Pérez-Montt

Avenida Santa Rosa 11735

La Pintana

Correo Postal Casilla 2 Correo 15

La Granja

Santiago

CHILE

Email: rolivare@uchile.cl

Recibido : 08-07-2010

Aceptado: 07-08-2010 